This item was submitted to Loughborough's Research Repository by the author.

Items in Figshare are protected by copyright, with all rights reserved, unless otherwise indicated.

\title{
HipHop als Hausmusik: Globale Sounds und (sub)urbane Kontexte
}

PLEASE CITE THE PUBLISHED VERSION

PUBLISHER

(C) Transcript-Verlag

VERSION

AM (Accepted Manuscript)

LICENCE

CC BY-NC-ND 4.0

REPOSITORY RECORD

Mager, Christoph, and Michael Hoyler. 2019. "Hiphop Als Hausmusik: Globale Sounds Und (sub)urbane Kontexte". figshare. https://hdl.handle.net/2134/6472. 
This item was submitted to Loughborough's Institutional Repository (https://dspace.lboro.ac.uk/) by the author and is made available under the following Creative Commons Licence conditions.

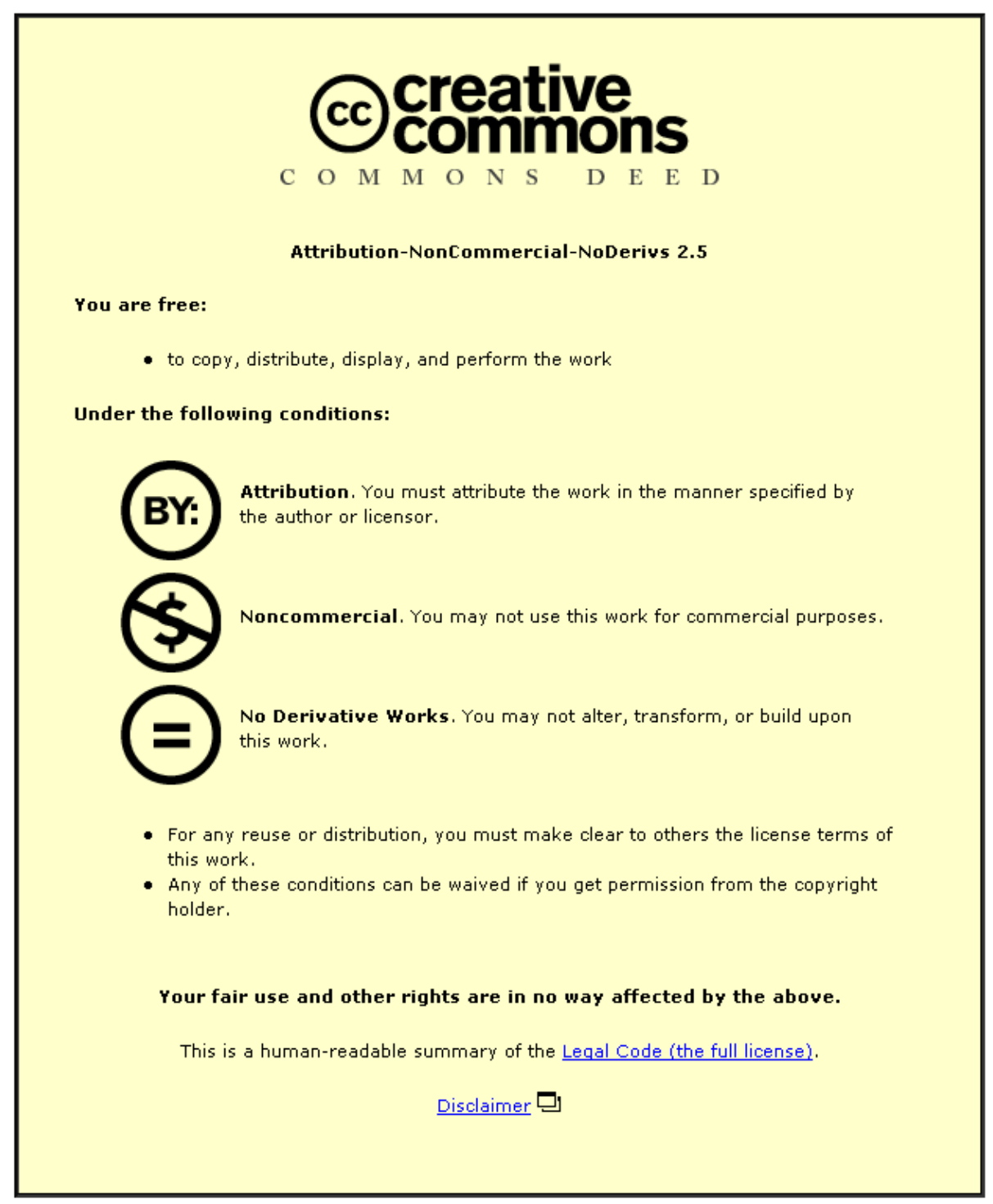

For the full text of this licence, please go to: http://creativecommons.org/licenses/by-nc-nd/2.5/ 


\title{
HipHop als Hausmusik: Globale Sounds und \\ (sub)urbane Kontexte
}

\author{
Christoph Mager und Michael Hoyler
}

\begin{abstract}
This paper explores the role of two constitutive spaces in the production and consumption of hip-hop music in Germany during the formative >Old Schook years from the mid-1980s to the early 1990s. Located in urban, suburban and rural contexts, public community centres and private bedrooms became important sites for the adoption and adaption of urban sounds rooted in US inner-city neighbourhoods. Whereas the former served as local homebases and trans-local nodes of wider hip-hop networks that brought young hip-hop artists together, the latter provided spaces of retreat and experimentation that gave room to the creation of alternative urban sounds.
\end{abstract}

\section{Einleitung: HipHop, Musik und die Geographien der Stadt}

Rap-Musik wird in der Regel, anderen musikalischen Genres wie Urban Blues oder Punk nicht unähnlich, in städtischen Kontexten verortet. Der Zusammenhang von Stadt und Sound ist dabei eng an die sozialen, ökonomischen und politischen Entstehungsbedingungen der jeweiligen musikalischen Formen geknüpft (Chambers 1985; Kloosterman 2005). Die verschiedenen musikalischen Praktiken im Rap gründeten sich während der Entstehungszeit von HipHop in den 1970er und 1980er Jahren auf die Alltagserfahrungen vor allem afroamerikanischer und hispanischer Jugendlicher in den durch Deindustrialisierung, städtebaulichen Niedergang und sozialräumliche Segregation gekennzeichneten New Yorker Stadtteilen Harlem und Bronx. Auf der Straße, in Parks, kleinen Clubs, community centers oder auf highschool-Parties präsentierten die frühen HipHop-DJs eine rhythmisch betonte und basslastige Musik, die sie mit Hilfe zweier 
Plattenspieler und einem Audiomixer aus verschiedenen Teilen älterer Tonträger zusammen mischten. Begleitet vom rhythmisierten und auffordernden Sprechgesang der MCs oder Rapper diente die Musik zunächst als Soundtrack für die städtischen Tanzböden und konnte bald zu einer zentralen Ausdrucksform des politischen und sozialen Kommentars von Minoritäten in der US-amerikanischen Stadt werden (Toop 2000; Keyes 2002: 17-66).

Auch auf klanglicher Ebene verweist Rap-Musik auf ihre städtische Herkunft. Regelmäßig finden urbane Klangphänomene Eingang in die Musik, wenn bspw. Konversationen auf der Straße, Sirenen, Schüsse, quietschende Autoreifen, Kindergeschrei oder der Klang von zerberstendem Glas als Teil der Rap-Ästhetik einkomponiert werden. Musiktechnologien wie Sequenzer, Drumcomputer und Sampler erlauben die Reproduktion der Klänge städtischer Umwelt im Aufnahmestudio und vervielfältigen die Möglichkeiten ihrer Wiedergabe, Manipulation und musikalischen Reorganisation. RapMusiker anerkennen die urbanen Dynamiken als kulturellen Kontext, aus dem sie ihr kreatives Potential schöpfen. Die Instabilität und Multilinearität spezifisch urbaner Lebenserfahrungen finden so ihre Entsprechung in den rhythmischen Flüssen, Schichtungen und Fragmentierungen der Musik. In diesem Sinne lässt sich Rap interpretieren als ein Produkt afroamerikanischer urbaner Kulturen und als aktueller »schwarzer« Sound der USamerikanischen Großstadt (Rose 1994: 21-61).

Seit in verschiedenen Teilen der Welt HipHop und Rap-Musik als hybride Formen kulturellen Ausdrucks Verbreitung fanden, werden auch dort städtische Symbole genutzt, um das Urbane zu repräsentieren und vor allem visuell zu inszenieren (Klein/Friedrich 2003: 85-140). Metropolitane Häusersilhouetten, Großwohnsiedlungen, heruntergekommene Straßenzüge oder mit Graffiti besprühte Wände sind gängige visuelle Markierungen populärer Bildmedien im HipHop auf globaler Ebene, die über einen Verweis zurück zur Ursprungserzählung eine lokale Authentifizierung von Musik, Musikern und Hörern sicherstellen sollen. Mehr als andere zeitgleiche Formen populärer Musik machte Rap für viele Jugendliche auch als politischer 
Kommentar der eigenen Situation Sinn. Daneben sind es aber gerade auch die klanglichen Eigenschaften von Rap-Musik, welche ihre Attraktivität in unterschiedlichen Rezeptionskontexten ausmacht. Der treibende musikalische Fluss, die rhythmische Flexibilität der Soundschichten und die Verwerfungen innerhalb der melodischen Progressionen üben motorische, körperlich-stimulierende Wirkungen aus, welche urbane Erfahrungen affektiv kommunizierbar werden lassen. In diesen raum-zeitlich transgressiven Eigenschaften von Rap als urbaner Musik liegt ein Schlüssel zum Verständnis von HipHop in den unterschiedlichsten Rezeptionskontexten (Walser 1995: 208-211; Frith 1996: 236).

Der vorliegende Beitrag fragt, ob und wie vor dem Hintergrund der kulturellen Signifikanz von Großstadt und ihren verschiedenen Rhythmen im USamerikanischen HipHop »Stadt« in anderen geographischen Kontexten musikalisch gefasst wurde. Die ausdrückliche Betonung eines räumlichen Bewusstseins und eines identitätsstiftenden Ortsbezugs wird dabei als eine der entscheidenden Eigenschaften von HipHop weltweit identifiziert. Deutlicher als in anderen Genres populärer Musik dienen diese Räumlichkeiten im HipHop als die zentralen Organisationsprinzipien von Bedeutungen, Werten und Handlungen (Forman 2000; Mager 2007). Ein besonderer Stellenwert kommt den konkreten Orten zu, an denen die Musik produziert wird. Über die Positionierung an spezifischen Orten und die Abgrenzungen gegenüber anderen Orten werden Respekt, Ansehen und Glaubwürdigkeit verhandelt und Authentizität immer wieder neu hergestellt. Durch die Identifizierung räumlicher Schlüsselkonstruktionen und zentraler physischer, sozialer und musikalischer Orte wird in diesem Beitrag die kulturelle Bedeutung verschiedener urbaner und suburbaner Räume bei der Konstituierung von HipHop in Deutschland untersucht. Das Herausarbeiten der spezifischen räumlichen Bedingungen der Rezeption und Produktion von Rap-Musik in West- und Ostdeutschland zu einem frühen Zeitpunkt will die geographischen Verschiebungen musikalischer Praktiken nachzeichnen und dabei einen Beitrag zum Verständnis der lokalen Adoption und Adaption einer global und urban konzipierten Form der populären Musik leisten (Bennett 2000: 52-70; Mitchell 2001; Androutsopoulos 2003). 
Zunächst wird kurz die soziale, ethnische und geographische Diversität der Rezeption von Rap-Musik in Deutschland gegen Ende der 1980er und zu Beginn der 1990er Jahre aufgezeigt, die eine einfache und unidirektionale Vermittlung der US-amerikanischen Formen in Frage stellt und den Blick auf die konkreten Orte der musikalischen Produktion und Reproduktion des urbanen Sounds lenkt. Der Hauptteil fokussiert auf zwei spezifische Räumlichkeiten, die sich als entscheidend erwiesen haben für die geographische und musikalische Formierung des Genres in Deutschland: Jugendzentrum und Jugendzimmer dienten und dienen als durch musikalische Praktiken konstituierte Orte, an denen urbane HipHop-Sounds auf unterschiedliche Weise konsumiert und (re)produziert werden.

\section{HipHop in Deutschland: Die Diversität der 1980er Jahre}

Die Anfänge von HipHop in Deutschland werden in der Regel mit der Ausstrahlung US-amerikanischer Filme wie Wild Style (1982), Beat Street oder Breakin' (beide 1984) in Fernsehen und Kino parallelisiert (Elflein 1998: 256; Verlan/Loh 2000: 60). In mehr oder weniger gelungener Form wurden hier einige der Kontexte dokumentiert, aus denen HipHop entstanden war, und zugleich Blaupausen dafür geliefert, was MCs sagten und DJs taten, wie Graffiti-Bilder aussehen konnten und welche Tanzschritte zu Rap-Musik möglich waren. Insbesondere Wild Style gilt als das zentrale Artefakt, welches HipHop als eine Art ganzheitliche Kulturform aus musikalischen, bildenden und darstellenden Elementen nach Westdeutschland kommunizierte. Der Film zeichnet sich durch eine sehr klare urbane Ästhetik aus, die verfallene Teile des New Yorker Stadtteils Bronx als städtebaulichen und sozialen Hintergrund für Graffiti präsentiert und den Soundtrack des Films immer wieder mit verschiedenen Rhythmen der Stadt verschmelzen lässt. Wie auch in anderen Ländern war Rap aus den USA in Deutschland zunächst nicht viel mehr als die Musik zum spektakulären Breakdance, der um 1984 mit großem medialen Interesse einen modischen Höhepunkt erlebte. Mit nachlassendem Enthusiasmus für die Tanzformen wendeten sich viele Jugendliche anderen Formen populärer Kultur zu, und es blieb nur eine kleine Subkultur interessierter Fans zurück, die sich punktuell und weit verstreut in urbanen, 
suburbanen und ruralen Kontexten nach und nach ein sachkundiges kulturelles Wissen auf Grundlage der frühen HipHop-Filme und eigener musikalischer Experimente im Amateurbereich erarbeiten konnten (Jacob 1993: 207-209).

Ende der 1980er Jahre machte die weitere Ausdifferenzierung der USamerikanischen Formen in Subgenres wie Reality Rap mit ausdrücklich politischen Inhalten und einer stärkeren Orientierung an Rock und Hardcore an der Ostküste sowie mit selbstbewusst zur Schau getragenen GangsterAttitüden und Synthesizer geschwängerten Funkeinflüssen an der Westküste die Musik für Jugendliche unterschiedlicher nationaler und ethnischer Herkunft auch in Deutschland attraktiv. Nachdem bereits etliche griechische, jugoslawische oder türkische Gastarbeiterkinder und Jugendliche aus anderen Migrationskontexten in den deutschen Großstädten zu den frühen Breakdancern zählten, nutzte diese Generation Plattenspieler, Mikrofon und Sampler, um eigene und hybride Rap-Versionen beispielsweise von Westküstenfunk oder New Jack Swing zu produzieren (Loh/Güngör 2002: 91103; El-Tayeb 2003). Für sie bot sich damit eine Möglichkeit, sich sowohl der Kultur der Elterngeneration als auch einer multikulturellen Integration in die deutsche Gesellschaft zumindest teilweise zu entziehen und sich als Teil verstärkt transnational verflochtener Gemeinschaften zu verstehen, die als diasporische Kulturen musikalische Verbindungen in die USA, nach Deutschland und in die jeweiligen Heimatländer aufweisen (Kaya 2001).

Die Vermittlung von Film, Fernsehen, Printmedien und Musikindustrie spielte zwar eine entscheidende Rolle bei der flächenhaften Verbreitung von RapMusik in Deutschland, aber es gab auch persönliche Kontakte in die USA oder zu Angehörigen der in Deutschland stationierten US-amerikanischen Streitkräfte, die zum Teil bereits zu Beginn der 1980er Jahre sehr lokal einen direkteren Zugang zu Rap-Musik ermöglichten. Von besonderer Bedeutung für die Produktion von HipHop-Musik war der Zugang zu Clubs, Läden für Musiktechnologie sowie raren Importplatten im Umfeld der Streitkräfte. Neben Berlin, Heidelberg, München und Stuttgart nahm zunächst Frankfurt eine herausragende Rolle ein. Durch Konzerte und DJ-Sets von New Yorker 
Künstlern im Umfeld der Airbase in Frankfurt lernten einige der Protagonisten Rap dort zu einem frühen Zeitpunkt als »schwarze« Musik eines multiethnischen, transatlantischen und explizit urbanen Raumes persönlich kennen. Frankfurt entwickelte sich zum ersten Zentrum einer eigenen HipHop-inspirierten Musikproduktion in Deutschland, als ab Mitte der 1980er Jahre deutsch-amerikanische Gruppen wie Bionic Force oder Snap und Künstler wie Moses P Platten vorlegten, die bereits nach US-amerikanischem Vorbild in professionellen Tonstudios eingespielt wurden (Cutmaster GB, persönliches Interview, Frankfurt, Mai 2002).

Dass die Konsumtion und Produktion von Rap-Musik aber keineswegs nur in den urbanen Zentren an der Spitze der Hierarchie des deutschen Städtesystems stattfand, zeigt ein Blick auf die räumliche Verteilung der Künstler mit Rap-Veröffentlichungen auf Tonträgern zu Beginn der 1990er Jahre (Mager 2003). Um 1990 erschienen erste Platten von Gruppen aus Berlin, Köln und Düsseldorf, gefolgt von Produktionen aus Nürnberg, Stuttgart und Hamburg sowie ergänzt durch eine wachsende Zahl von Kompilationen mit Künstlern verschiedener lokaler Herkunft. Bereits 1993 lässt sich kein eindeutiges Zentrum von HipHop-Musik in Deutschland mehr ausmachen. Auffällig sind Produktionen aus Städten und Gemeinden der mittleren Hierarchiestufe des deutschen Städtenetzes wie Braunschweig, Bielefeld, Lüdenscheid oder Ulm. Konsumtion und Produktion von HipHop-Musik in Deutschland war damit von Anfang an nicht ausschließlich an die Zentren der großen Städte gebunden, sondern fand im suburbanen oder gar, wie das Beispiel der frühen Produktionen von L(egally) S(pread) D(ope) belegt, im rural-peripheren Kontext der Eifel statt (RickSki, persönliches Interview, Köln, November 2001).

Die räumliche und zeitliche Diversität des Adoptions- und Adaptionsprozesses von Rap-Musik in Deutschland wird besonders deutlich, wenn die Verhältnisse in Ostdeutschland mit berücksichtigt werden. Zwar begann auch in der DDR mit einem US-Film das jugendliche Interesse an HipHop und insbesondere an Breakdancing, dass aber Beat Street weniger als Unterhaltung, sondern als politische Dokumentation der Lebensverhältnisse 
der afroamerikanischen Bevölkerung in den USA in das Kinoprogramm aufgenommen wurde, zeigt bereits, wie die grundlegenden ideologischen und sozioökonomischen Systemunterschiede zwischen Ost- und Westdeutschland den Grad der Offenheit gegenüber US-amerikanischer populärer Musik prägen konnten (Connell/Gibson 2003: 104-105). Die relativ isolierte Position der DDR gegenüber kulturellen Flüssen des Westens und die eingeschränkte Reisefreiheit machten es Jugendlichen fast unmöglich, an Informationsmaterial oder HipHop-Tonträger zu kommen. Hinzu traten die spartanische technische Ausstattung des Durchschnittshaushalts und die mangelhaften Kenntnisse der englischen Sprache, welche Rap-Musik in der DDR kaum wahrnehmbar werden ließ (Wagner 1999).

Trotz einer großen Diversität musikalischer Praktiken in unterschiedlichen sozialen, politischen und ökonomischen Kontexten der Adoption und Adaption von HipHop lassen sich einige wenige Räumlichkeiten identifizieren, denen eine zentrale Rolle in der Formierungsphase von Rap-Musik in West- und Ostdeutschland zugesprochen werden kann. Rap als urbaner Sound wurde in Deutschland über urbane, suburbane und rurale Raumkategorien hinweg vornehmlich in den konstituierenden Kontexten der häuslichen Jugendzimmer und der translokalen Jams der Alten Schule in den Jugendzentren konsumiert, produziert und reproduziert.

\section{HipHop-Sounds als urbane Hausmusik?}

Die frühe Entwicklung von Rap-Musik in Deutschland lässt sich allgemein fassen als der schrittweise Übergang von der Imitation US-amerikanischer Modelle - bis in die späten 1980er Jahre wird häufig in englischer Sprache über die instrumentals der B-Seiten importierter US-Platten gerappt (Jacob 1993: 206-209) - über »eingedeutschte« Versionen in den späten 1980er und frühen 1990er Jahren zu zunehmend hybriden und re-ethnifizierten Adaptionen, die stärker auf eigene musikalische Kenntnisse und Vorlieben sowie auf die persönliche Alltagswelt Bezug nehmen (Larkey 1993: 89-203; Regev 1997). War Rap für die Jugendlichen zunächst »die letzte Besatzermusik«, welche ganz selbstverständlich als neue und spannende 
Form einer amerikanisierten Popularkultur konsumiert, imitiert und adaptiert werden wollte (Niemczyk 1999), erfolgte recht rasch der Übergang vom Konsumieren und Mitrappen einzelner Passagen zu Versuchen, das Gehörte mit den vorhandenen Mitteln zu kopieren und die auffälligsten Ungereimtheiten kreativ auszuräumen. Torch, der heute als eine der zentralen Figuren der Entwicklung von HipHop in Deutschland gilt, erinnert sich an seine ersten eigenen Rap-Sounds:

»Ich habe viele Schallplatten gehört, hatte sehr viel musikalischen Input. 1979 erstes Kurtis Blow-Album und auch viele GoGo-Platten. Irgendwann - gerade bei Rap kommt das schnell auf - willst du nicht nur die Melodie kennen. Und irgendwann kanntest Du die Texte auswendig, von jedem Lied, das du mitsingst. Was besonders ist bei Rap: die Rapper nennen ihre Namen, es ist etwas sehr Persönliches. Es gibt keinen Kurtis Blow-Song, in dem er nicht kurz sagt >Kurtis Blow<. [...] Und immer wenn >Kurtis Blow< kam, wollte ich natürlich meinen Namen einsetzen. Nur, der Text hat sich nicht mehr gereimt, und dann musste ich ihn umschreiben. [Musikalisch] angefangen hat's damit, dass man einen Kassettenrekorder hatte. Mein Vater hatte ein Doppelkassettendeck, mit dem konnte ich Sachen loopen. Das heißt, ich habe irgendwo eine Passage gehört, die mir gefallen hat und hab' sie einfach immer wieder hin kopiert. Und manchmal hat's genau gesessen, das hab' ich dann meinen ganzen Freunden vorgespielt. Und so hat man ein eigenes Instrumental geschaffen, worüber ich dann rappen konnte. Und nach dem Kassettenrekorder hat man am Plattenspieler versucht, irgendwie zu scratchen. [...] Und dann hab' ich irgendwann mal von meinem Musiklehrer ein Effektgerät für Gitarren ausgeliehen, anschließend hab' ich mir einen Drumcomputer gekauft, den kleinsten und günstigsten. Dann hat meine Mutter eine alte Gitarre gehabt, an der zwei Saiten gefehlt haben. Wir haben uns einfach was zurechtgesponnen, alles Mögliche, was einen Ton erzeugt, alles: Beatbox, Human Beatbox mit dem Mund. Und wir haben das alles zusammengeschmissen und haben einfach Musik gemacht, so gut wie's ging. Einfach alles Mögliche, scheißegal, Hauptsache es kommt irgendwo ein Ton raus. [...] Dann haben [wir] unsere ersten selbst geschriebenen Texte drüber probiert. Wir haben dann schon recht früh, '87, die Band gegründet, 
Advanced Chemistry« (Torch, persönliches Interview, Heidelberg, Juli 2001).

Von ähnlichen Versuchen, durch musikalische Experimente oder das möglichst exakte Wiederholen von aus den HipHop-Filmen memorierten Sequenzen eine Musik zu erzeugen, welche die grundlegenden Eigenschaften von Fluss, Schichtung und Bruch aufwies und sich mit den eigenen Hörerfahrungen deckte, berichten viele der frühen Protagonisten von Rap in Deutschland. Charakteristisch war dabei eine ausgeprägte Do it yourself-Mentalität der Amateurmusiker, die sich nicht zuletzt durch ein geringes oder teures Angebot an Informationen, Technologien und geeigneten musikalischen Ausgangsmaterialien immer auch durch den Austausch mit anderen auszeichnete. In der zweiten Hälfte der 1980er und in den frühen 1990er Jahren fanden diese musikalischen Praktiken insbesondere an zwei Orten statt, die sich als prägend für die frühe Entwicklung von Rap-Musik in West- und Ostdeutschland erwiesen: die öffentlichen Jugendzentren und die privaten Jugendzimmer.

\subsection{Von Jugendzentren...}

»Zehn Jahre her, da waren wir in deiner Stadt.

Wo ist heut' die crowd, die damals so gejubelt hat? Haben darauf geachtet, dass der Text 'nen Sinn hat. Ich verlang nicht viel, nur dass ihr euch daran erinnert.

Aus Jux treten wir noch manchmal auf im JuZ, wenn 'ne andere Band nicht kann vor zwanzig Mann, aber wen juckt's?« (Torch featuring Toni L der Pate: »Wir waren mal Stars« [360\%/V2, 2000]).

Bürgerhäuser, soziokulturelle Einrichtungen und Jugendzentren gehören seit rund 40 Jahren zur kulturellen Ausstattung vieler deutscher Städte und Gemeinden. In Westdeutschland sollte die kulturelle Arbeit dieser Einrichtungen zunächst drei in den 1960er Jahren deutlich wahrnehmbaren Krisen begegnen. Zum einen identifizierte eine neue Kulturpolitik die Unterscheidung zwischen Hoch-, Volks- und Populärkultur als überkommen und versuchte, die traditionellen Kulturtempel für breite Bevölkerungskreise zu 
öffnen. Unter dem Schlagwort »Demokratisierung der Kultur« sollte jedem Bürger Chancengleichheit beim Zugang zu kulturellen Ressourcen ermöglicht werden. Eng verbunden mit dieser Forderung nach »Kultur für alle« war der Versuch, einer persistenten, sozioökonomisch bestimmten Schieflage der Bildungsbeteiligung durch wohlfahrtsstaatliche Reformen entgegenzuwirken. »Bildung für alle« sollte das Problem einer gerechteren Verteilung des gesellschaftlichen Wohlstands lösen helfen. Der neue kulturpolitische Impetus reagierte schließlich auch auf eine Krise der Stadt, die, so die Mutmaßung, mit dem Wiederaufbau nach Ende des Zweiten Weltkriegs nach rein funktionalen Gesichtspunkten restrukturiert worden sei, und in dieser Form zunehmend zu sozialer Entfremdung, Isolation und Apathie der Bewohner geführt habe (Mitscherlich 1965; Glaser/Stahl 1974). Die Aufgabe der Stadtplanung war es dann, neue Spielräume zu eröffnen, in denen die erweiterten Begriffe von Kultur und Bildung vermittelt und mit Leben erfüllt werden konnten. Ein Ergebnis dieser Ideen war in den Folgejahren die Etablierung von Jugendzentren und Bürgerhäusern in ganz Westdeutschland, die teilweise unter Anleitung von Sozial- und Kulturarbeitern nichtkommerzielle und kreative Eigenbetätigung fördern und im Stadtteil oder der Gemeinde ethnisch und sozial integrativ wirken sollten (Freytag/Hoyler/Mager 2002).

Ab etwa 1986 begannen viele der HipHop-Interessierten, diese öffentlichen Räume für ihre musikalischen Praktiken und den Austausch mit anderen zu nutzen. In Jugendzentren und Bürgerhäusern konnten sich in der Folgezeit lokal lose HipHop-Gemeinschaften bilden, deren jugendliche Mitglieder außerhalb der direkten Kontrolle von Elternhaus und Erwachsenenwelt ihrer eigenen Vorstellung von Band und populärer Musik nachgehen konnten. Mark Terkessidis beschreibt 1991 in der Musikzeitschrift Spex beispielhaft die zeitgenössische Situation im Nürnberger Stadtteil Gostenhof, wo

»im Laufe der letzten Jahre türkische, italienische, halbperuanische und deutsche Homeboys zu einer der größten, produktivsten und politisch radikalsten HipHop-Communities in Deutschland zusammengewachsen sind. Im Gostenhofener Jugendzentrum regiert Hardcore-Rap so wie anderswo 
Hardcore-Rock. Die amtierenden Könige sind Incredible Al und Chill Fresh a.k.a. King Size Terror. [...] Die Homebase all dieser Musiker ist das Jugendfreizeitheim Gostenhof, wo es tatsächlich einen Raum für DJ's gibt, in dem sich immer alle treffen; die Basic-Einrichtung hat die Stadt hingestellt, Plattenspieler, Sampler und das restliche Equipment haben sie sich selbst zusammengekauft« (Terkessidis 1991: 39).

Die Rhetorik des Musikjournalisten ist geprägt von den US-amerikanischen HipHop-Diskursen der Zeit. Begriffe wie homebase und homeboy verweisen auf den geographischen Ursprungsort von HipHop in den Großstädten der USA. Der Untertitel des Artikels »South Central Gostenhof« rückt Nürnbergs Südstadt in die Nähe des notorischen Stadtteils South Central in Los Angeles und weckt Assoziationen von Ganggewalt, Kriminalität und urbaner Halbwelt. Dieser Eindruck wird verstärkt durch ein illustrierendes Photo, auf dem einige ausnahmslos männliche Mitglieder grimmig dreinblickend vor einem heruntergekommenen Gebäude posieren und das Bild einer segregierten, vernachlässigten und verfallenden Stadtlandschaft evozieren. Trotz dieser vereinfachenden Parallelisierung des Städtischen in unterschiedlichen räumlichen Kontexten und einer eingeschränkten Vergleichbarkeit der Erfahrungen »schwarzer« und hispanischer Jugendlicher in den Ghettos USamerikanischer Innenstädte mit dem Altag der Jugendlichen in Städten und Gemeinden europäischer Wohlfahrtsstaaten wird deutlich, wie wichtig der Gedanke einer base called home für das Verständnis der sozialen und klanglichen Organisation von populärer Musik im Allgemeinen und von Rap im Besonderen ist (Leyshon/Matless/Revill 1998). Rap-Musiker »repräsentieren«, sie stehen regelmäßig für ihren sozialen und musikalischen Ort, der zugleich für sie stehen soll (Forman 2000). Auch in bundesdeutschen Kontexten wurde und wird der Produktionszusammenhang von Rap häufig durch eine starke Verbundenheit mit dem lokalen Umfeld und einer Gruppe von Gleichgesinnten charakterisiert. In der Anfangszeit der musikalischen Konsumtion und Produktion von Rap-Musik in Deutschland ermöglichten vor allem die Jugendzentren, persönliche Hörerfahrungen mit anderen zu teilen, sich über die Fortschritte der musikalischen Do it yourself-Versuche und RapFähigkeiten auszutauschen oder die eigene Musik vor einem kleinen 
Publikum in Live-Situationen zu präsentieren. Homebase drückt das Gefühl der Verbundenheit mit einer solchen Lokalität aus, an der sich Freunde und Bekannte treffen, um gemeinsam zu musizieren und Musik zu konsumieren.

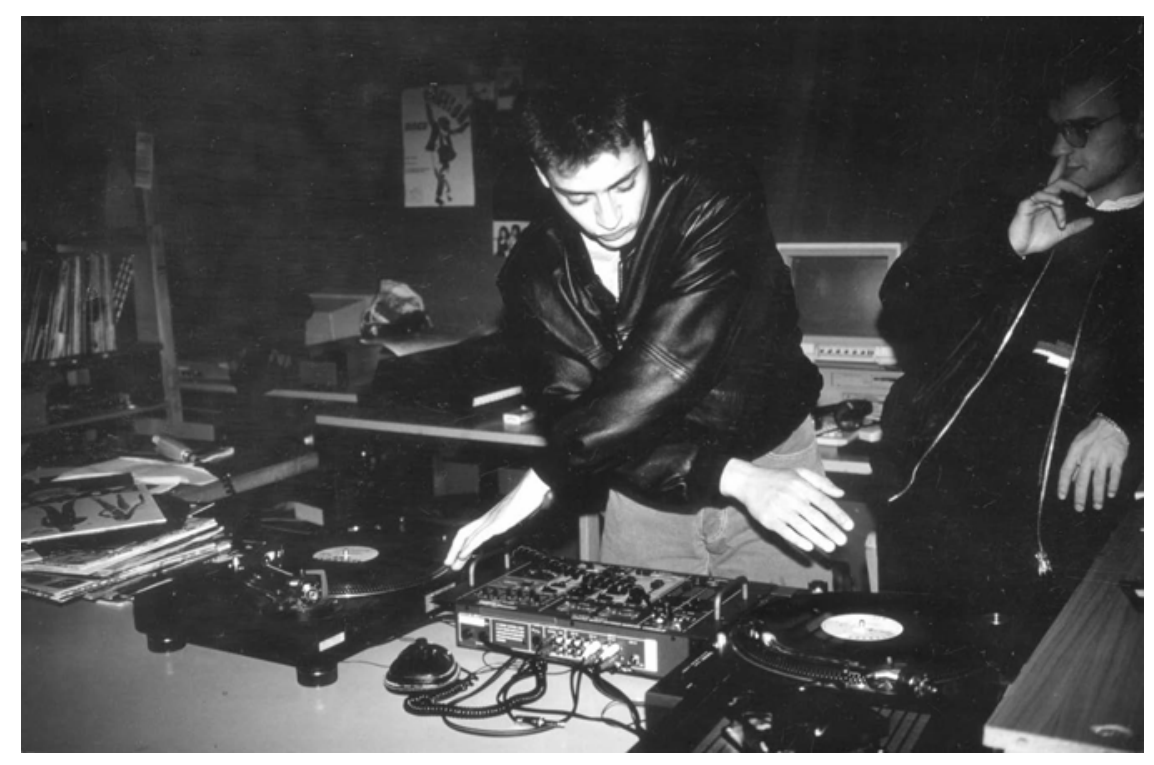

Abbildung 1: Das Jugendzentrum als ein entscheidender Ort der frühen Konsumtion und Produktion von Rap-Musik in Deutschland. Mitglieder der türkisch-deutschen Rap-Gruppe Karakan üben zu Beginn der 1990er Jahre im Nürnberger Jugendhaus Gostenhof DJ-Techniken (Abdruck mit freundlicher Genehmigung des Kinder- und Jugendhauses Gostenhof).

Verlief die frühe Rap-Entwicklung in verschiedenen Städten und Gemeinden Westdeutschlands zunächst relativ isoliert, entstand bei den Amateurmusikern bald das Bedürfnis, sich mit anderen auszutauschen und die verschiedenen musikalischen Aktivitäten zu vernetzen. Linguist, Mitglied der Heidelberger Formation Advanced Chemistry, blickt auf diese dynamischen Entwicklungen zurück:

»[Das Jugendhaus] wurde später noch einmal sehr wichtig, als wir anfingen, uns in Deutschland etwas breiter zu machen. Als Advanced Chemistry gegründet wurden und wir angefangen haben, auf Jams zu spielen, fanden die ersten Jams in den Jugendhäusern statt oder wurden von Jugendhäusern organisiert. Das war dann später, so gegen 1987/88, als wir mobiler wurden« (Linguist, zit. n. Loh/Güngör 2002: 131). 
Linguist führt hier den Gedanken eines mobilen HipHop-Raumes ein, der durch Mobilität und Austausch zwischen verschiedenen Orten konstituiert wird. Auf der Basis von persönlichen Kontakten oder Informationen aus Zeitungen brachen die frühen HipHop-Aktivisten an den Wochenenden meist per Bahn auf, um an anderen Orten in Deutschland jene Musiker aufzusuchen, die ihre Begeisterung für Rap teilten. Graffiti und die eigenwilligen Kleidungsstile der Protagonisten waren zuverlässige Indikatoren für HipHop-Enthusiasten, die sich zudem in aller Regel in einem der Jugendzentren vor Ort finden ließen. Hier wurden Adressen ausgetauscht, wechselten Kassetten den Besitzer oder konnten zukünftige gemeinsame Aktivitäten vereinbart werden. Auf Grundlage dieser gegenseitigen Besuche entstanden nach und nach informelle Netzwerkstrukturen, die Jugendliche nicht nur aus verschiedenen lokalen urbanen und suburbanen Kontexten in Deutschland polyzentrisch verbanden, sondern auch in andere westeuropäische Länder wie Schweiz, Italien, Frankreich, Niederlande oder Dänemark reichten. Gelegentlich wurden deutschland- oder europaweite Jams der Alten Schule veranstaltet, HipHop-Parties, bei denen vielleicht 50 bis 200 jugendliche HipHop-Enthusiasten aus unterschiedlichen nationalen, ethnischen und sozioökonomischen Zusammenhängen in einer der homebases zusammenkamen, um in einer, häufig aber in mehreren der HipHop-Disziplinen Rapping, DJing, Breakdancing und Graffiti aktiv mitzuwirken und zu wettstreiten (Verlan/Loh 2000: 102-110). Hier verschob sich der Fokus der Aufmerksamkeit ständig, sodass jeder zu allen HipHopPraktiken einen Beitrag leisten konnte. Die übliche Unterscheidung von Künstler und Publikum verschwamm ebenso wie die scharfe Trennung von Bühne und Zuschauerraum.

Auf den Jams fand, einer Messe nicht unähnlich, ein reger Austausch von musikalischem Wissen und stilistischen Ideen statt, der an Lernprozesse durch Beobachtung anderer geknüpft war. Die Freestyle-Wortgefechte der Rapper und die Beats der DJs waren hier an performative Akte innerhalb einer temporären Gemeinschaft gebunden. Musikalisch stand das Spiel mit schnellen Reimen und der Spaß an der technisch-handwerklichen Seite von HipHop im Vordergrund: sampling, scratching, cutting, rapping. Die Qualität 
der Musik und die Sorgfalt ihrer Produktion konnten dabei aus mehreren Gründen von untergeordneter Bedeutung bleiben. Eine grundlegende Eigenschaft der Musik, welche für die Jam-Situation produziert oder ausgewählt wurde, war eine relative Einfachheit und die leichte kontextsensitive Adaptionsfähigkeit der Sounds. So musste es für die teilweise wechselnden DJs möglich sein, die unter Umständen relativ schnellen Verschiebungen der Aufmerksamkeit beispielsweise von den Breakdancern über die DJs hin zu den Rappern mit wenigen Handgriffen musikalisch zu meistern. Waren die Breakdancer auf tanzbare Sounds und Rhythmen angewiesen, die ihren verschiedenen Stilen wie breaking, popping oder locking Rechnung tragen konnten, präsentierten die DJs ihre Fingerfertigkeit und musikalischen Vorlieben als dynamische und abwechslungsreiche Soundsets. Die freestyle sessions hingegen, bei denen sich mehrere Rapper am Mikrofon abwechseln und sich gegenseitig durch immer ausgefeiltere Reime zu übertreffen versuchen, bedürfen endloser, eher monotoner und tendenziell schnörkelfreier Sounds, die sich, ohne auf einen Höhepunkt zuzulaufen, an der Geschwindigkeit und am Rhythmus des normalen Sprechens orientieren. So ist beispielsweise in der Abschlusssequenz der ZDF-Dokumentation Lost in Music von 1993 eine freestyle session auf der Ostberliner Insel der Jugend zu Beginn der 1990er Jahre dokumentiert, deren Sound sich hauptsächlich an der Funktion eines monotonen Metronoms für die Rapper orientiert.

Eine weitere Beschränkung komplexer und ausgefeilter Sound(re)produktion auf den Jams war die vorhandene technische Ausstattung der Jugendzentren und Veranstaltungsorte. Konfigurationen, die üblicherweise den Anforderungen von Rockkonzerten genügten, konnten einem aufwendigen DJ-Set, einer zusätzlichen Live-Instrumentation oder dem Einsatz komplexer Musiktechnologie im Wege stehen. Neben der Unsicherheit, was genau die Anreisenden an Mischpulten, Verstärkern und Lautsprechersystemen erwartete, war auch häufig unklar, welche musikalischen Präferenzen des lokalen und überlokalen Publikums auf den Jams anzutreffen waren. Da sich aufgrund der geringen absoluten Zahl von Rap-Musikern zunächst kaum ausgeprägte stilistische Soundcharakteristika einzelner Städte oder lokal 
organisierter Produktionszusammenhänge herausbilden konnten Ausnahmen sind vielleicht die stark vom englischen Britcore beeinflussten Hardcore-Produktionen in Hamburg und Bremerhaven -, traf man auf den Jams »die unterschiedlichsten Stile an: klassische B-Boys, Hypedancer, Miami-Bass-Freaks, West-Coast-Fans und so weiter« (Loh/Güngör 2002: 98). Der Kontext war dabei nie notwendigerweise urban, viele der Jams fanden in kleineren Städten wie Aschaffenburg, Bruchsal, Gießen und Lüdenscheid oder in Vororten statt.

Auch in der DDR standen seit den 1960er Jahren mit den Kulturzentren der Volkseigenen Betriebe oder den öffentlichen Klubs der Massenorganisationen dezentrale staatliche Kultureinrichtungen zur Verfügung. Aufgrund der strengen inhaltlichen Ausrichtung an zentral verordneten Leitlinien der einheitlichen sozialistischen Kultur- und Sozialpolitik, einer strikten Ablehnung US-amerikanischer Populärkultur und einer rigiden Überwachung aller kulturellen Aktivitäten blieb hier nur wenig Raum für selbstbestimmte kulturelle Praktiken. Erst im Verlauf der 1980er Jahre war es jungen Menschen zunehmend möglich, eigene musikalische und subkulturelle Ideen zu verwirklichen und die politischen Räume der kulturellen Infrastruktur subversiv als Freiräume lokaler Musikszenen zu nutzen (Wicke 1998). Neben Rockmusik wurden in den Einrichtungen auch die Aktivitäten der verhältnismäßig wenigen HipHop-Musiker geduldet, die sich aufgrund der mangelnden Möglichkeiten des Austauschs mit anderen Musikern aber kaum vernetzen oder öffentlich wahrgenommen werden konnten. Einige der ab 1990 nach westdeutschem Vorbild nach und nach (wieder)eröffneten Jugendund Kulturhäuser erfüllten in der Folgezeit auch für ostdeutsche Rap-Musiker ähnliche Funktionen wie im Westen. Hier standen teilweise einfache Aufnahmemöglichkeiten zur Verfügung, und einzelne Jugendzentren wie das Conne Island in Leipzig wurden in den regelmäßigen Jam-Kalender der Alten Schule aufgenommen (Hoyler/Mager 2005).

Die nationalen und internationalen Netzwerke der Alten Schule fanden von Zeit zu Zeit in den Räumlichkeiten der Jugendhäuser und Kulturzentren ephemere Bodenhaftung. HipHop-Jams waren damit weniger lokal 
abgegrenzte und sozial abgeschlossene Räume, sondern müssen unter Berücksichtigung der komplexen Beziehungen auf verschiedenen Maßstabsebenen als relationale musikalische Orte gefasst werden, die sich durch das spezifische Zusammentreffen von unterschiedlichen Akteuren, Einflüssen und Kontakten konstituieren (Massey 1998).

\title{
$3.2 \ldots$ und Jugendzimmern
}

\author{
»Ach, ich glaube ich kenne keinen, \\ der sich so viel Mühe gemacht hat, \\ ein Kinderzimmer zu gestalten.« \\ (Kinderzimmer Productions: »Intro« [EFA, 1996])
}

Neben den musikalischen Netzwerken der Alten Schule, die in den öffentlichen Jugendhäusern und Kulturzentren der west- und später auch der ostdeutschen Städte und Gemeinden sowohl ihre Basis als auch ihre temporären Knoten fanden, existierten weitere Orte, an denen Konsumtion und Produktion von Rap-Musik stattfand. Viele der Übungsstunden und musikalischen Experimente mit Plattenspielern und Mikrofon fanden nach wie vor in den privaten Räumen der Jugendzimmer und Wohnhauskeller im Osten wie im Westen statt, obgleich aus zum Teil unterschiedlichen Gründen.

Wenn sich die Mitglieder der Formation Die Drei Rüben aus Ulm zu Beginn der 1990er Jahre in Kinderzimmer Productions umbenennen, so illustriert dies die enge Bindung der musikalischen Praktiken an die intimen Räume der eigenen vier Wände. Für die Gruppe war »ein stinknormales Kinderzimmer« in einer der ubiquitären suburbanen Reihenhaussiedlungen Deutschlands der Ort, an dem sich DJ Quasimodo und Rapper Textor nachmittags trafen, um zusammen Musik zu hören und zu produzieren. Textor beschreibt im Interview die musikalische Herangehensweise von Kinderzimmer Productions zu Beginn der 1990er Jahre:

»Wir waren tatsächlich auch so lange im Kinderzimmer deswegen, weil wir so qualitätsbewusst waren. Uns war einfach klar, dass das, was wir selber 
fabrizieren, nicht an das ranreicht, was die amerikanischen Vorbilder abliefern. Und wir wollten mit Hilfsmitteln etwas präsentieren, das zumindest auf eine eigene Art und Weise mithalten kann. Also es ging schon ganz stark auf das Entwickeln einer persönlichen Identität und eines persönlichen Ausdrucks. Und wir dachten am Anfang auch, dass es der ganzen deutschen HipHop-Szene darum gehen würde. Ging's am Anfang auch mehr oder weniger, das driftete dann später immer weiter auseinander. Und die ganze Jam-Szene hat zu großen Teilen den Eindruck vermittelt, es ist egal, ob es nicht so gut ist, Hauptsache man macht was. Und da konnten wir nicht mithalten, da gab's einfach einen Reflex dagegen, wo man gesagt hat: >Ne, das ist einfach noch nicht gut genug. Wenn ich mich auf 'ne Bühne stelle, will ich, dass das gut ist! « Und dann waren wir mit Ulm natürlich auch weit ab vom Schuss« (Textor, persönliches Interview, Heidelberg, November 2005).

Textor betont hier ein Verständnis von Rap-Musik, das einen deutlichen Kontrast zur Soundproduktion der Jam-Szene darstellt. Rap-Musik ist für Kinderzimmer Productions nicht zuallererst begleitender Soundtrack für Breakdancer oder freestyle sessions auf einer HipHop-Party in irgendeinem Jugendzentrum, sondern Ergebnis des Versuchs, sich über ein ergründendes Hören und Konsumieren dem Produktionsprozess der US-amerikanischen Vorbilder zu nähern und auf dieser Grundlage teils imitierende, teils neue und dennoch vergleichbare eigene Sounds zu kreieren. Die Musik der Vorbilder von Kinderzimmer Productions - mit Public Enemy, Gang Starr, De La Soul oder A Tribe Called Quest ausschließlich Künstler der US-amerikanischen Ostküste, die manchmal unter dem Signet College Boy oder Conscious Rap zusammengefasst werden - war von Anfang an gedacht als eine Art Blaupause, auf deren Grundlage »Band gespielt«, die einzelnen Schritte musikalischer Produktion nachgestellt und HipHop-Techniken im Privaten für sich selbst noch einmal neu erfunden werden konnten. Solch musikalische Praxis konnte nicht am sozialen Ort der HipHop-Jam im Jugendzentrum stattfinden, sondern war zunächst zurückgeworfen auf einen intimen Rückzugsort, der das Studium von Musikzeitschriften, das Ergründen von Samples und das möglichst originalgetreue Wiederholen der Musik aus New York ermöglichte. Aus diesen »qualitätsbewussten« Produktionskontexten 
gehen dann auch viele der frühen HipHop-Tonträger in Deutschland hervor. Insofern meint Textors Bemerkung, Kinderzimmer Productions in Ulm hätten »weit ab vom Schuss« gelegen, weniger eine räumliche Distanz zu den Zentren der Jam-Aktivitäten der Alten Schule als einen symbolischen Abstand von den dominierenden Prozessen in den Jugendzentren und den Ökonomien der Alten Schule.

Auch in der DDR verbrachten viele junge Leute trotz des breiten Angebotes an dezentralen staatlichen Kultureinrichtungen große Teile ihrer Freizeit allein oder zusammen mit Freunden in den eigenen vier Wänden, wo Spiele gespielt, Musik gehört oder ferngesehen wurde - häufig westdeutsche Programme. Für die wenigen HipHop-begeisterten Jugendlichen wurden diese Privatzimmer zu Freiräumen außerhalb der direkten staatlichen Kontrolle und der Überwachung durch ein System von Informanten. In kleinen Heimstudios konnten mit Hilfe von Plattenspielern und simpler Kassettentechnologie einfache Stücke jenseits der staatlichen Produktionsund Distributionsmonopole realisiert, aufgenommen und vervielfältigt werden (Krekow/Steiner 2000: 114). 


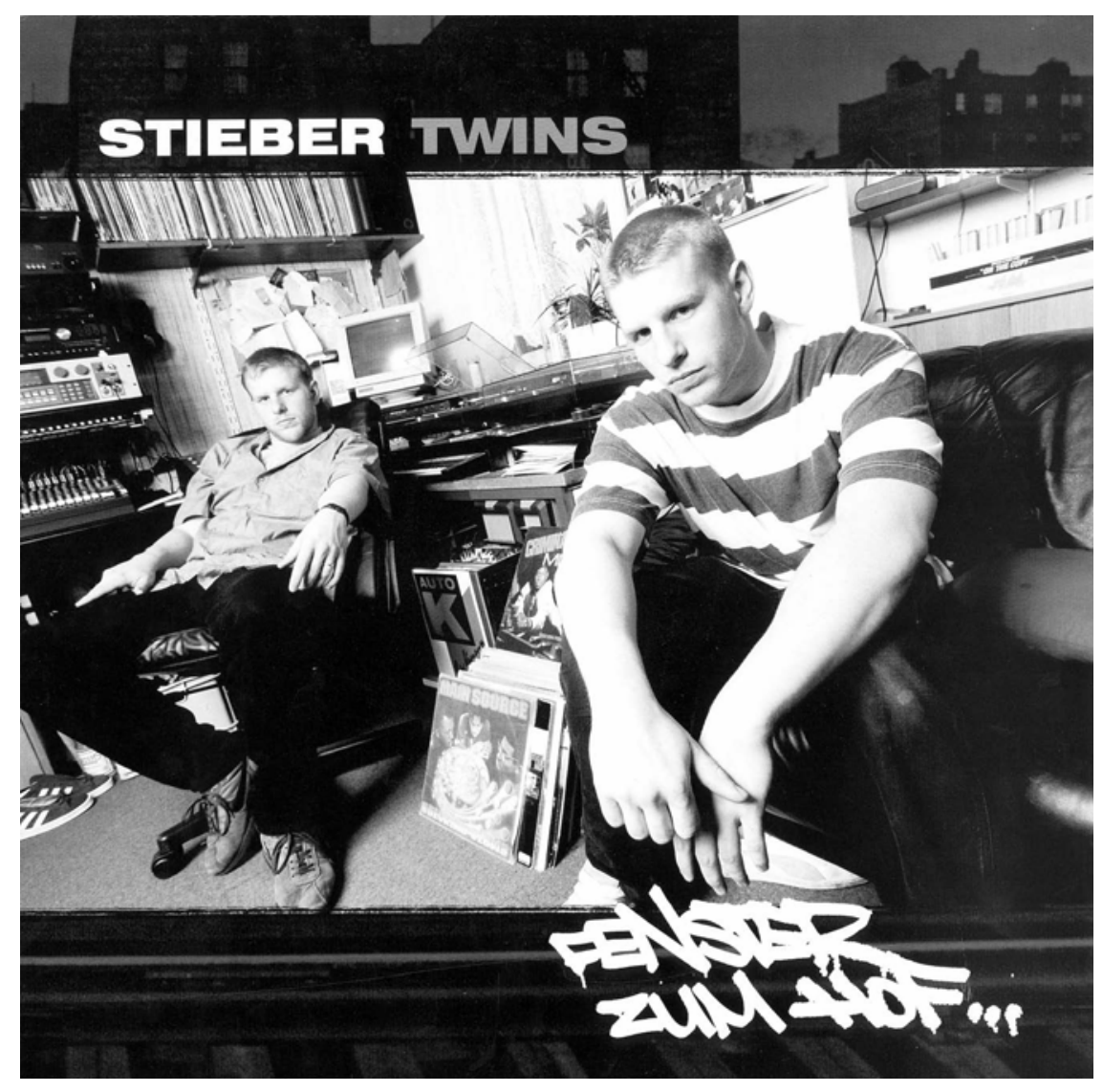

Abbildung 2: Das Jugendzimmer als Studio für Rap-Musik. Die Stieber Twins aus Heidelberg posieren für das Album Fenster zum Hof in ihrem zum Aufnahmestudio umfunktionierten Zimmer (Abdruck mit freundlicher Genehmigung von MZEE Productions).

Dass Jugendzentrum und Jugendzimmer keine sich gegenseitig ausschließenden Räumlichkeiten der Rap-Produktion in Deutschland darstellen, soll ein weiteres Beispiel belegen. Abbildung 2 zeigt das Cover der Langspielplatte Fenster zum Hof, welche die Stieber Twins 1996 beim Label MZEE veröffentlichten. Die Brüder präsentieren sich in ihrem zum Aufnahmestudio umfunktionierten Zimmer im elterlichen Haus komplett mit Gardinen und Zimmerpflanzen im Heidelberger Stadtteil Wieblingen, wo jeder Zentimeter freien Raumes durch miteinander vernetzte Gerätschaften wie Plattenspieler, Mischpult, Sampler, Computer und andere elektronische Musikgerätschaften nutzbar gemacht worden ist. Das heimische Jugendzimmer ist auch hier der Ort musikalischer Konsumtion und Produktion - das Home Alone Studio -, in dem sich die beiden Musiker für die 
Fotoaufnahme selbstbewusst und entspannt nach getaner Arbeit präsentieren und den sie repräsentieren wollen. Mit der Inszenierung der Photographie verbunden sind musikalische Referenzen, die mit der Platte Criminal Minded von Boogie Down Productions, Breaking Atoms von Main Source und dem aus einer halb geöffneten Schublade herauslugenden Soundtrack zu Wild Style zu den Anfängen von HipHop nach New York City und auf das Goldene Rap-Zeitalter der späten 1980er und frühen 1990er Jahre an der USamerikanischen Ostküste verweisen. Zugleich bilden städtische Häuserruinen am oberen und unteren Rand des Covers den städtischen Hintergrund, vor dem die Musik verstanden werden will. Obwohl auch hier das private Jugendzimmer im Mittelpunkt der musikalischen »bedroom production« steht, weisen die Gastauftritte von Rappern aus Aachen, Minden oder Köln über den engen Kontext der häuslichen Tüfteleien hinaus und verorten die Musik der Stieber Twins innerhalb eines nachhaltigen sozialen Netzwerks der Alten Schule, das in Jam-Besuchen in den 1980er und frühen 1990er Jahren wurzelt (Szillius 2005).

\section{Fazit: Urbane Sounds und (sub)urbane Kontexte}

»HipHop in Deutschland ist [...] auch ein Kleinstadtphänomen.«

(DJ Rabauke, zit. n. Krekow/Steiner 2000: 184)

Der Blick auf die Formierungsphase von Rap-Musik in West- und Ostdeutschland während der zweiten Hälfte der 1980er und zu Beginn der 1990er Jahre legt eine Vielzahl von Adoptions- und Adaptionsprozessen urbaner, US-amerikanischer Popularmusik offen. In vielen Städten, Gemeinden und Dörfern begannen Jugendliche unterschiedlicher ethnischer, räumlicher und sozioökonomischer Herkunft, HipHop-Sounds zu konsumieren und zu (re)produzieren. Für Deutschland wurden in dieser Studie zwei zentrale Räumlichkeiten der Konsumtion und Produktion identifiziert, die eine entscheidende Rolle für die geographische und musikalische Organisation von Rap-Musik spielten: die öffentlichen Jugendzentren und die privaten Jugendzimmer. Während die Zentren als homebases und ephemere Knoten im translokalen Netzwerk von HipHop-Enthusiasten der Alten Schule 
fungierten, dienten Jugendzimmer als Rückzugsorte des Musikhörens und des musikalischen Experimentierens, welche Raum für die authentische Kreation alternativer Formen urbaner Sounds boten. HipHop-Musik in den Jugendzentren war der Soundtrack einer sozialen Zusammenkunft, der Jam, bei der eine Einheit der verschiedenen HipHop-Disziplinen so zelebriert wurde, wie es die frühen Filme vermittelt hatten. Über die imaginierte Nähe der Jugendzentrums-Szene zum Do it yourself-Sound und zur zentralen urbanen Ursprungserzählung von HipHop aus den USA stellen die Angehörigen der Alten Schule bis heute soziale und musikalische Authentizität her. Die Tüfteleien im deutschen Jugendzimmer zielten dagegen zuallererst auf die Imitation und technische Reproduktion der Sounds aus den USA in privaten Rückzugsräumen jenseits eines umfassenden sozialen Gemeinschaftserlebnisses. Hier stellte sich Authentizität vor allen Dingen musikalisch her über die qualitative hochwertige Wiedergabetreue urbaner Sounds. Jugendzentrum und Jugendzimmer in West- und Ostdeutschland dienten und dienen in urbanen, suburbanen und ruralen Kontexten als durch musikalische Praktiken konstituierte Orte, an denen urbane HipHop-Sounds auf unterschiedliche Weise konsumiert und produziert werden und für die der HipHop-Sound zu einem Stück Hausmusik geworden ist.

\section{Literatur}

Androutsopoulos, Jannis (2003). »Einleitung.« In: HipHop. Globale Kultur lokale Praktiken (= Cultural Studies 3). Hg. v. dems. Bielefeld: transcript.

Bennett, Andy (2000). Popular Music and Youth Culture. Music, Identity and Place. Basingstoke u.a.: Macmillan. Chambers, lain (1985). Urban Rhythms. Pop Music and Popular Culture. London u.a.: Macmillan.

Connell, John / Gibson, Chris (2003). Sound Tracks: Popular Music, Identity and Place. London u.a.: Routledge.

El-Tayeb, Fatima (2003). „If You Can't Pronounce My Name, You Can Just Call Me Pride. Afro-German Activism, Gender and Hip Hop.« In: Gender \& History 15, S. 460-486.

Elflein, Dietmar (1998). »From Krauts With Attitudes to Turks With Attitudes. Some Aspects of Hip-Hop History in Germany.« In: Popular Music 17, S. 
255-265.

Forman, Murray (2000). »Represent. Race, Space and Place in Rap Music.« In: Popular Music 19, S. 65-90.

Freytag, Tim / Hoyler, Michael / Mager, Christoph (2002). »Soziokultur und ihre Zentren.« In: Nationalatlas Bundesrepublik Deutschland. Band 6: Bildung und Kultur. Hg. v. Institut für Länderkunde. Heidelberg u.a.: Spektrum Akademischer Verlag, S. 118-119.

Frith, Simon (1996). Performing Rites. On the Value of Popular Music. Cambridge, MA: Harvard University Press.

Glaser, Hermann / Stahl, Karl Heinz (1974). Die Wiedergewinnung des Ästhetischen. Perspektiven und Modelle einer neuen Soziokultur. München: Juventa.

Hoyler, Michael / Mager, Christoph (2005). »HipHop ist im Haus. Cultural Policy, Community Centres and the Making of Hip-Hop Music in Germany.« In: Built Environment 31, S. 237-254.

Jacob, Günther (1993). Agit-Pop. Schwarze Musik und weiße Hörer. Texte zu Rassismus und Nationalismus, HipHop und Raggamuffin. Berlin u.a.: Edition ID-Archiv.

Kaya, Ayata (2001). Sicher in Kreuzberg. Constructing Diasporas. Turkish Hip-Hop Youth in Berlin. Bielefeld: transcript.

Keyes, Cheryl L. (2002). Rap music and street consciousness. Urbana u.a.: University of Illinois Press.

Klein, Gabriele / Friedrich, Malte (2003). Is it real? Die Kultur des HipHop. Frankfurt/M.: Suhrkamp.

Kloosterman, Robert C. (2005). „Come Together. An Introduction to Music and the City.« In: Built Environment 31, S. 181-191.

Krekow, Sebastian / Steiner, Jens (2000). Bei uns geht einiges - die deutsche Hip-Hop-Szene. Berlin: Schwarzkopf \& Schwarzkopf.

Larkey, Edward (1993). Pungent Sounds. Constructing Identity with Popular Music in Austria (= Austrian Culture 9). New York: Lang.

Leyshon, Andrew / Matless, David / Revill, George (1998). »Introduction. Music, Space, and the Production of Place.« In: The Place of Music. Hg. v. Andrew Leyshon, David Matless und George Revill. London u.a.: Guilford Press, S. 1-30. 
Loh, Hannes / Güngör, Murat (2002). Fear of a Kanak Planet: HipHop zwischen Weltkultur und Nazi-Rap. Höfen: Hannibal.

Mager, Christoph (2003). »1, 2, 3... from New York to Germany. Raum, Repräsentation und Rap-Musik.« In: Frontiers of Geography. Hg. v. Ákos Jakobi und József Nemes Nagy. Budapest: Department of Regional Geography, Eötvös Loránd University, S. 199-212.

Mager, Christoph (2007). HipHop, Musik und die Artikulation von Geographie. Stuttgart: Steiner.

Massey, Doreen (1998). »The Spatial Construction of Youth Cultures.« In: Cool Places. Geographies of Youth Cultures. Hg. v. Tracey Skelton und Gill Valentine. London u.a.: Routledge, S. 121-129.

Mitchell, Tony (2001). »Introduction. Another Root - Hip-Hop outside the USA.« In: Global Noise. Rap and Hip-Hop Outside the USA. Hg. v. Tony Mitchell. Middletown, CT: Wesleyan University Press, S. 1-38.

Mitscherlich, Alexander (1965). Die Unwirtlichkeit unserer Städte. Frankfurt/M.: Suhrkamp.

Niemczyk, Ralf (1999). Die Fantastischen Vier. Die letzte Besatzermusik. Die Autobiographie. Köln: Kiepenheuer \& Witsch (2. Aufl.).

Regev, Motti (1997). »Rock Aesthetics and Musics of the World.« In: Theory, Culture \& Society 14 , S. 125-142.

Rose, Tricia (1994). Black Noise. Rap Music and Black Culture in Contemporary America. Hanover, NH: Wesleyan University Press.

Szillius, Stephan (2005). „Stieber Twins. Zwei Namen ein Titel.« In: Juice Sonderheft 1, S. 24-25.

Terkessidis, Mark (1991). »Kingsize Terror. Breakbeats und Klassenkampf im Zeichen der Burg. South Central Gostenhof.« In: Spex 9, September, S. 3840.

Toop, David (2000). Rap Attack 3. African Rap to Global Hip Hop. London u.a.: Serpent's Tail (3. Aufl.).

Verlan, Sascha / Loh, Hannes (2000). 20 Jahre HipHop in Deutschland. Höfen: Hannibal.

Wagner, Mike (1999). »Rap is in the house. HipHop in der DDR.« In: Wir wollen immer artig sein... Punk, New Wave, HipHop, Independent-Szene in der DDR 1980-1990. Hg. v. Ronald Galenza und Heinz Havemeister. 
Berlin: Schwarzkopf \& Schwarzkopf, S. 314-325.

Walser, Robert (1995). »Rhythm, Rhyme, and Rhetoric in the Music of Public Enemy.«In: Ethnomusicology 39, S. 193-217.

Wicke, Peter (1998). »Popmusik in der DDR. Zwischen Anpassung und Widerstand.« In: but I like it. Jugendkultur und Popmusik. Hg. v. Peter Kemper, Thomas Langhoff und Ulrich Sonnenschein. Stuttgart: Reclam, S. 268-283. 